\title{
PRODUTIVIDADE DAS CARACTERÍSTICAS AGRONÔMICAS DA MAMONEIRA COM EFEITO DE ADUBAÇÃO ORGÃNICA E FERTILIZANTES QUÍMICOS EM CATOLÉ DO ROCHA-PB
}

\author{
PRODUCTIVITY FEATURES WITH EFFECT AGRONOMICAL OF CASTOR \\ BEAN ORGANIC FERTILIZER CHEMICALS AND FERTILIZERS ON THE \\ CATOLÉ DO ROCHA-PB
}

\section{PRODUCTIVIDAD DE AGRONÓMICO DE MAMONA FUNCIONES CON EFECTO DE LA FERTILIZACIÓN EN FERTILIZANTES QUÍMICOS Y ORGÁNICOS CATOLÉ DO ROCHA-PB}

\author{
José Sebastião MELO FILHO ${ }^{1}$ \\ Valdeci Andrade DANTAS ${ }^{2}$ \\ Diego Frankley da Silva OLIVEIRA ${ }^{3}$ \\ Leonardo Pereira da SILVA ${ }^{4}$ \\ Fabiana Xavier COSTA ${ }^{5}$
}

\begin{abstract}
RESUMO: Objetivou-se com este trabalho analisar a produtividade da mamoneira, após adubação com resíduos sólidos orgânicos (casca de pinhão manso) e doses crescentes de nitrogênio e fixas de fósforo nas condições edafoclimáticas. O experimento foi desenvolvido na área experimental do Campus IV da Universidade Estadual da Paraíba, no município de Catolé do Rocha - PB. Os tratamentos consistiram de uma combinação fatorial com duas formas de utilização de casca de pinhão manso (natural e moída). Na quantidade de $3 \mathrm{t} / \mathrm{ha}^{-1} \mathrm{e}$ três doses de nitrogênio $\left(0,30,60 \mathrm{Kg} / \mathrm{ha}^{-1}\right)$, aplicado no cultivo em delineamento experimental de blocos ao acaso. Os resultados para as variáveis estudadas obtiveram-se efeito significativo em função de diferentes doses de nitrogênio na fitomassa do caule, fitomassa da raiz principal, número de sementes do cacho e a fitomassa de 100 sementes do cacho. Sendo que os maiores valores obtidos foi com a dose de $60 \mathrm{~kg} / \mathrm{ha}^{-1}$, superando respectivamente as demais quantidades de 30 e $0 \mathrm{~kg} / \mathrm{ha}^{-1} \mathrm{~N}$. Foi constatado que nenhuma das variáveis estudadas sofreu efeito significativo para fator tipo de casca.
\end{abstract}

Palavras-chave: produção; fitomassa; nitrogênio

\footnotetext{
1 Mestre em Sistemas Agroindustriais - UFCG. Universidade Federal de Campina Grande. Campus Pombal PB. Centro de Ciências e Tecnologia Agroalimentar. Sistemas Agroindustriais. sebastiaouepb@yahoo.com.br 2 Universidade Estadual da Paraíba. Departamento de Agrárias e Exatas. Ciências Agrárias. valdeci.emepa@bol.com.br

3 Universidade Estadual da Paraíba. Departamento de Agrárias e Exatas. Ciências Agrárias. diegofrankley.g3@gmail.com

4 Universidade Estadual da Paraíba. Departamento de Agrárias e Exatas. Ciências Agrárias. leonardopereira1992@gmail.com

5 Prof. Dra. do Departamento de Agrárias e Exatas da Universidade Estadual da Paraíba - UEPB/Campus IV. Bióloga, Doutorado em Recursos Naturais. CEP 58884-000 - Catolé do Rocha - Paraíba - Brasil. fabyxavierster@gmail.com
} 


\begin{abstract}
The objective of this work to analyze the yield of castor bean, after fertilization with organic solid waste (jatropha bark) and increasing levels of nitrogen and phosphorus fixed at conditions. The experiment was conducted at the Campus IV of the experimental area of the State University of Paraíba, in Catolé do Rocha - PB. Treatments consisted of a factorial combination with two ways of using jatropha peel (natural and ground). In the amount of $3 \mathrm{t} / \mathrm{ha}-1$ and three nitrogen rates $(0,30,60 \mathrm{~kg} / \mathrm{ha}-1)$, applied in cultivation in experimental design of randomized blocks. The results for the studied variables were obtained significant effect due to the application of nitrogen in stem biomass, biomass of the main root, number of cluster seeds and biomass of 100 seeds of the bunch. The highest values obtained was with the dose of $60 \mathrm{~kg} / \mathrm{ha}-1$, respectively outperforming the other quantities of 30 and 0 $\mathrm{kg} / \mathrm{ha}-1 \mathrm{~N}$. It was found that none of the studied variables suffered significant effect factor for type of shell.
\end{abstract}

Key words: Production; biomass; nitrogen

RESUMEN: El objetivo de este trabajo para analizar el rendimiento de la semilla de ricino, después de la fertilización con residuos sólidos orgánicos (corteza jatropha) y el aumento de los niveles de nitrógeno y fósforo en las condiciones fijadas. El experimento se llevó a cabo en el Campus IV de la zona experimental de la Universidad del Estado de Paraíba, en Catolé do Rocha - PB. Los tratamientos consistieron en una combinación factorial con dos formas de utilizar la cáscara de jatropha (planta natural y). En la cantidad de $3 \mathrm{t} / \mathrm{ha}-1 \mathrm{y}$ tres dosis de nitrógeno $(0,30,60 \mathrm{~kg} /$ ha-1), aplicado en el cultivo en el diseño experimental de bloques al azar. Los resultados para las variables estudiadas se obtuvieron efectos significativos debido a la aplicación de nitrógeno en la biomasa del tallo, la biomasa de la raíz principal, número de semillas de racimo y la biomasa de 100 semillas del montón. Los valores más altos obtenidos fue con la dosis de $60 \mathrm{~kg} / \mathrm{ha}-1$, respectivamente, superando las otras cantidades de $30 \mathrm{y} 0 \mathrm{~kg}$ / ha-1N. Se encontró que ninguna de las variables estudiadas sufrió factor de efecto significativo para el tipo de concha.

Palabras clave: producción; la biomasa; nitrógeno

\title{
Introdução
}

A cultura da mamona (Ricinus communis L.) conquistou seu espaço econômico, político e ambiental no Brasil devido ao interesse pela indústria rícino química e pela busca de novas fontes de energias, visto que obtenção de diesel a partir do petróleo tem custo elevado, além da queima deste combustível ser altamente poluente. Os resultados apontam o biodiesel como uma das alternativas viáveis a substituir combustíveis obtidos do petróleo. Assim, a mamona vem ganhando espaço em todo o território nacional, principalmente nos estados do Nordeste e Centro Oeste (MORO, 2008). 
Esta planta é de fácil cultivo e resistente à escassez de água, por isso é ideal para o extenso semiárido brasileiro. Nessa região existem quase quatro milhões de hectares apropriados, onde se pode alcançar o rendimento de até 1,5t de sementes por hectare, enquanto a média nacional é de $750 \mathrm{~kg}$ de bagalha quilos. Além disso, a mamona pode se converter em uma alternativa produtiva para cerca de 1,5 milhões de pessoas que passam necessidades na região mais pobre do Brasil (SILVA, LINO, 2009). É uma planta que se desenvolve em regiões tropicais e semiáridas, mas apesar de ser resistente a seca para produzir bem, a mamona necessita de pelo menos 16 nutrientes e aproximadamente $500 \mathrm{~mm}$ de chuva bem distribuída ao longo de seu ciclo (COSTA et al., 2009; BELTRÃO et al., 2008).

A qualidade da água é um dos fatores que ocasionam efeito negativo no desenvolvimento das culturas e afetam a produção, visto que a água é constituinte dos tecidos vegetais, chegando até mesmo construir mais de $90 \%$ de algumas plantas; desta forma e para a utilização da água de qualidade inferior na agricultura, deve-se utilizar um manejo racional, através de alternativas economicamente viáveis, de modo que a cultura desenvolva a produtividade esperada (MEDEIROS et al., 2007).

A mamoneira carece de informações sobre tecnologia para fertilização do solo, pois há poucos relatos na literatura sobre seu comportamento em diferentes condições como cultivares, níveis de fertilidade do solo, clima, disponibilidade de água etc. Porém, sabe-se que esta planta é exigente em fertilidade e que é possível aumentar sua produtividade pelo adequado fornecimento de nutrientes através da adubação (FERREIRA et al., 2005; SEVERINO et al., 2005). O fósforo destaca-se como o elemento mais estudado na adubação da mamoneira e com maior resposta em produtividade (LAVRES JUNIOR et al., 2009). Depois do nitrogênio, o fósforo é o elemento que mais limita o crescimento dos vegetais na maioria dos solos (MARENCO e LOPES, 2009).

A casca subproduto obtido através do descascamento dos frutos da mamoneira, que representa em torno de 30 a $40 \%$ do total do cacho, quantitativo importante na matriz da produção, pode e deve ser aproveitado como adubo orgânico, pois apresenta níveis razoáveis de constituintes inorgânicos, totalizando $2,5 \%$ do seu total, cujo valor é muito próximo ao da polpa que é de $3,1 \%$, portanto adequado para a recuperação de terras esgotadas do semiárido, constituindo-se em uma fórmula de aproveitamento importante deste resíduo (COELHO, 2006).

O óleo extraído de suas sementes, é utilizado na produção de remédios, cosméticos, na construção civil, na indústria automobilística, no revestimento de poltronas e paredes de avião, na fabricação de plásticos biodegradáveis, na fabricação de tintas, vernizes, 
lubrificantes, vidros a prova de balas entre outros (SOUZA et al., 2009) e como matéria-prima para produção do biodiesel.

Desta forma objetivou-se com este trabalho avaliar a capacidade produtiva da mamona BRS energia em função de resíduos sólidos orgânicos (casca de pinhão manso muída e natural) e adubação mineral (doses crescentes de nitrogênio e fixas de fósforo), nas condições edafoclimáticas do município de Catolé do Rocha-PB.

\section{Material e métodos}

O experimento foi realizado na unidade experimental da Universidade Estadual da Paraíba Campus IV, no sitio Cajueiro município de Catolé do Rocha, em parceria com a Embrapa Algodão de Campina Grande-PB. O município fica a $272 \mathrm{~m}$ de altitude, 6²0’38’S de Latitude e $37^{\circ} 44^{\prime} 48^{\prime \prime}$ O de Longitude, localiza-se no Sertão Paraibano, ao Norte com Almino Afonso e Patú (RN) e parte do município de Belém do Brejo do Cruz; ao Sul com Jericó e Riacho dos Cavalos; a Leste com Brejo do Cruz e a Oeste com Brejo dos Santos e João Dias (RN). De clima quente e seco, cuja temperatura média anual é de $27^{\circ} \mathrm{C}$. Foram realizadas análises físicas e químicas do solo utilizado no plantio (Tabela 1 e 2).

Tabela 1-Características químicas (fertilidade) do solo que foi usado no experimento. UEPB, Catolé do Rocha - PB, 2011.

\begin{tabular}{lllllr}
\hline $\mathrm{pH} \mathrm{H}_{2} \mathrm{O}$ & Complexo Sortivo (meq/100g de solo) & $\%$ & $\%$ & $\%$ & $\mathrm{mg} /$ \\
\hline
\end{tabular}

\begin{tabular}{ccccccccccccc}
\hline$(1: 2,5)$ & $\mathrm{Ca}$ & $\mathrm{Mg}$ & $\mathrm{Na}$ & $\mathrm{K}$ & $\mathrm{S}$ & $\mathrm{H}+\mathrm{Al}$ & $\mathrm{T}$ & $\mathrm{CO}$ & $\mathrm{N}$ & $\mathrm{MO}$ & $\mathrm{P}$ \\
\hline 7,49 & 5,66 & 2,09 & 0,20 & 0,24 & 7,86 & 0,00 & & 7,86 & 0,61 & 0,06 & 1,05 & 2,57
\end{tabular}

Análises realizadas no Laboratório de Solo da Universidade Federal de Campina Grande. Campina Grande - PB, 2011.

Tabela 2 - Características físicas do solo que foi usado no experimento. UEPB, Catolé do Rocha - PB, 2011.

\begin{tabular}{|c|c|c|c|c|c|c|c|}
\hline \multicolumn{3}{|c|}{ Densidade $-\mathrm{kg} / \mathrm{dm}^{3}$} & \multicolumn{4}{|c|}{ Granulometria - \% } & \multirow[b]{2}{*}{ Classificação Textural } \\
\hline Global & Real & $\begin{array}{l}\text { Porosidade } \\
\text { Total }(\%)\end{array}$ & $\begin{array}{l}\text { Areia } \\
\text { Grossa }\end{array}$ & Areia Fina & Silte & Argila & \\
\hline 1,02 & 2,67 & 61,90 & 54,60 & 43,90 & 23,00 & 22,40 & $\begin{array}{l}\text { Franco Argilo } \\
\text { arenoso }\end{array}$ \\
\hline
\end{tabular}

Análises realizadas no Laboratório de Solo da Universidade Federal de Campina Grande. Campina Grande - PB, 2011. 
Os tratamentos consistiram de uma combinação fatorial de duas formas de utilização da casca de pinhão manso (natural e moída), na quantidade de 3 t/ha e três doses de Nitrogênio $\left(0,30,60, \mathrm{Kg} / \mathrm{ha}^{-1}\right)$,utilizado em Delineamento Experimental de blocos ao acaso em arranjo fatorial 2 × 3, sendo (2) representando duas formas de utilização da casca (natural e moída) e (3) as dosagens de Nitrogênio (0,30 e 60kg/ha), com 4 repetições, totalizando 24 parcelas, plantada em vasos plásticos de 60 L. Na tabela 3 encontra-se a análise química da casca de pinhão manso.

Tabela 3- Características químicas da casca de mamona que foi usada no experimento. UEPB, Catolé do Rocha - PB, 2011.

\begin{tabular}{|c|c|c|c|c|c|c|c|c|c|c|c|c|c|}
\hline Umid & PB & $\mathrm{CZ}$ & $\mathrm{N}$ & $\mathrm{P}$ & $\mathrm{P}_{2} \mathrm{O}_{5}$ & $\mathrm{~K}$ & $\mathrm{~K}_{2} \mathrm{O}$ & $\mathrm{C}$ & $\mathrm{CaO}$ & $\mathrm{Mg}$ & $\mathrm{MgO}$ & $\mathrm{S}$ & $\mathrm{MO}$ \\
\hline - --- & & & & & & & 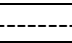 & & & & & & --- \\
\hline 7,50 & 11,93 & 12,61 & 1,91 & 0,12 & 0,97 & 4,61 & 5,54 & 1,27 & 1,78 & 0,30 & 0,53 & 0,17 & 87,39 \\
\hline
\end{tabular}

Análises realizadas no Laboratório de Química da Embrapa Algodão. Campina Grande - PB, 2011

O plantio foi realizado em vasos plásticos de $60 \mathrm{~L}$, tendo como medidas $57 \mathrm{~cm}$ de altura, $40 \mathrm{~cm}$ de diâmetro superior e $26,5 \mathrm{~cm}$ de diâmetro inferior, utilizando a cultivar BRS energia desenvolvido pela Embrapa Algodão.O cultivo foi realizado no primeiro semestre agrícola de 2011. A adubação nitrogenada foi aplicada toda na base. Foram semeadas 3 sementes por cova de mamona BRS Energia em cada vaso e aos 12 dias após a emergência das plântulas realizou-se o desbaste, mantendo-se uma planta por vaso.

A coleta dos dados foi realizada a cada quinze dias após a germinação, num total de seis períodos de coleta. As variáveis estudadas foram: fitomassa do cacho (MSC), fitomassa da raiz (MSR), número de sementes do cacho (NSCH), fitomassa de 100 sementes do cacho (PSSCH). Os dados das variáveis foram submetidos à análise de variância pelo teste $\mathrm{F}$ e comparadas através de análise de regressão a 1 e 5\% de probabilidade. A análise estatística foi realizada no software SAS.

\section{Resultados e discussão}

Os resumos das análises de variâncias na Tabela 4 demonstram que na fitomassa do cacho, não foi observado influência significativa pelo tipo de adubo casca natural e moída, obtendo influência significativa pelas doses de adubo químico e interação entre ambos, no entanto na fitomassa da raiz principal para o tipo de adubo casca não foi verificada a ocorrência significativa, já para o número de sementes do cacho e a fitomassa de 100 sementes do cacho em função de diferentes doses de nitrogênio foi verificada a ocorrência de 
significância para as doses de adubo químico e observou-se que nenhuma das variáveis estudadas sofreu efeito significativo para fator tipo de casca.

Tabela 4. Resumos das analises de variâncias (quadrados médios), referentes às variáveis de produção da mamona BRS energia: fitomassa do caule (FC), fitomassa da raiz principal (FR), número de sementes do cacho (NSCH), fitomassa de 100 sementes do cacho (F100SCH). UEPB, Catolé do Rocha - PB, 2011.

\begin{tabular}{|c|c|c|c|c|c|}
\hline \multirow[b]{2}{*}{ FV } & \multirow[b]{2}{*}{ GL } & \multicolumn{4}{|c|}{ 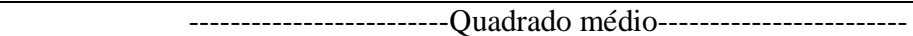 } \\
\hline & & $\mathrm{FC}$ & FR & $\mathrm{NSCH}$ & F100SCH \\
\hline Casca (C) & 1 & $78,84^{\text {ns }}$ & $10,27^{\mathrm{ns}}$ & $1426,04^{\mathrm{ns}}$ & $14,88^{\mathrm{ns}}$ \\
\hline Dose (D) & 2 & $2221,64 * *$ & $224,22 * *$ & $12146,79 * *$ & $677,98^{* *}$ \\
\hline $\mathrm{C} \times \mathrm{D}$ & 2 & $309,78^{\mathrm{ns}}$ & $35,56^{\mathrm{ns}}$ & $2372,54^{\mathrm{ns}}$ & $166,97^{\mathrm{ns}}$ \\
\hline Resíduo & 15 & 138,31 & 27,15 & 1137,05 & 59,14 \\
\hline Total & 31 & - & - & - & - \\
\hline Média Geral & & 10,23 & 4,5 & 29,34 & 6,69 \\
\hline $\mathrm{CV} \%$ & & 31,74 & 34,11 & 26,10 & 23,37 \\
\hline
\end{tabular}

Ilustra-se na figura 1 o comportamento para as variáveis de produção fitomassa do cacho da mamoneira BRS energia analisando a adubação orgânica com casca de mamona natural e moída em função de doses crescentes de nitrogênio $\left(0,30,60\right.$ e Kg/ha $\left.{ }^{-1}\right)$.

Onde a influência significativa da aplicação de doses crescentes de $\mathrm{N}$ no comportamento de produção da mamoneira para a variável fitomassa do caule (FC). Observase que houve um aumento linear nas crescentes doses de N. Os maiores valores obtidos com a dose de $60 \mathrm{~kg} / \mathrm{ha}^{-1}$.

Esse efeito positivo da adubação orgânica, fonte de matéria orgânica para o solo, corrobora com Bayer e Mielniczur (2008), quando afirmam que a matéria orgânica é um componente fundamental da capacidade produtiva dos solos, por causa dos seus efeitos sobre a disponibilidade de nutrientes, capacidade de troca de cátions, a complexação de elementos tóxicos e micronutrientes, a infiltração e a retenção de água.

A disponibilidade natural dos nutrientes do solo é determinada em virtude da natureza do seu material de origem, do clima e do manejo, mas mesmo sendo originalmente fértil, um solo poderá sofrer, ao longo do tempo, redução na sua disponibilidade de nutrientes (PROCHNOW e ROSSI, 2009). Neste sentido, é importante saber quanto adubar como adubar e quando adubar. 


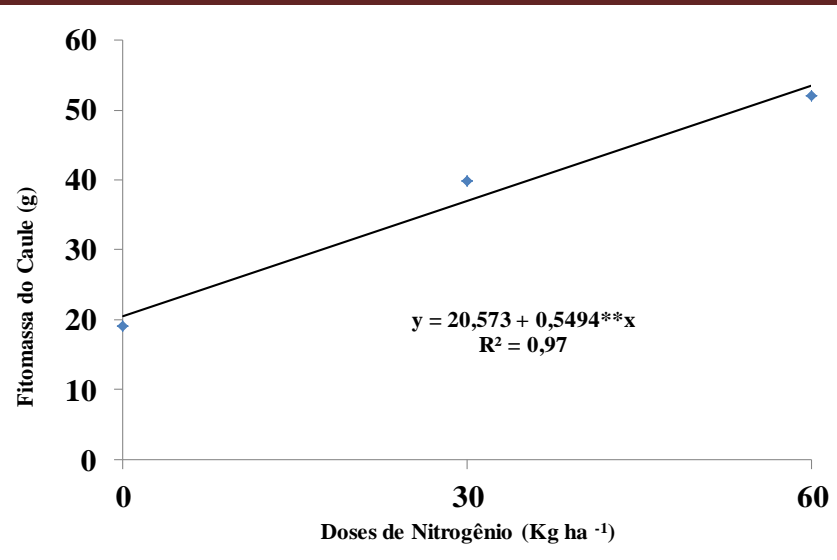

Figura 1. Modelos de regressão para fitomassa do caule da mamoneira BRS Energia com adubação orgânica: casca de mamona natural e moída em função de doses crescentes de Nitrogênio. Catolé do Rocha - PB, 2011.

Na figura 2 ilustra o comportamento para as variáveis de produção fitomassa da raiz principal da mamoneira BRS energia analisando a adubação orgânica, com casca de mamona natural e moída em função de doses crescentes de nitrogênio.Observa-se que a fitomassa da raiz principal é maior na dose de $60 \mathrm{~kg} / \mathrm{ha}^{-1}$ de N. Que superam respectivamente as demais quantidades de 30 e $0 \mathrm{~kg} / \mathrm{ha}^{-1}$ de $\mathrm{N}$.

A mamoneira é muito exigente em fertilidade do solo, tendo produtividade muito alta em solos com alta fertilidade natural ou que receberam adubação em quantidade adequada. Deve-se sempre fazer a análise de solo e fornecer a quantidade de fertilizantes recomendada pelo laudo técnico. Mesmo sob intenso déficit hídrico a mamoneira é capaz de aproveitar a adubação, o que diminui o risco dessa prática, principalmente em regiões semiáridas (SEVERINO et al,. 2005).

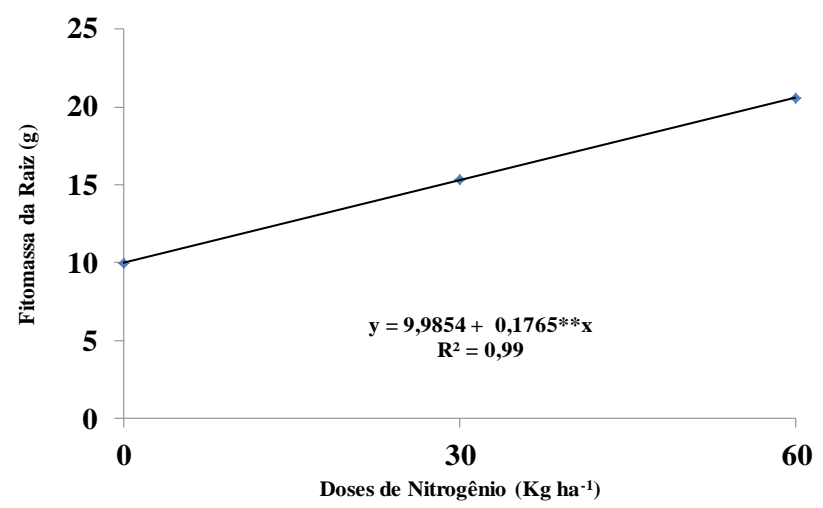

Figura 2. Modelos de regressão para fitomassa da raiz principal da mamoneira BRS Energia com adubação orgânica: casca de mamona natural e moída em função de doses crescentes de Nitrogênio. Catolé do Rocha - PB, 2011. 
De acordo com Lima et al. (2005), a casca, em sua forma primária, é um componente de origem orgânica que usado na adubação propicia a formação de macroporos no substrato que facilitam as trocas gasosas e a distribuição da água, no entanto, tem decomposição muito lenta, de forma que sua contribuição como material fornecedor de nutrientes é pouco significativa.

A casca de mamona além de apresentar quantidades significativas de N, P e K, favorece a melhoria das propriedades físicas e químicas do solo, atuando no aumento da capacidade de armazenamento de água, aeração, elevação do pH pela redução da acidez do solo e outros (SEVERINO et al. 2006).

A adubação é uma das principais técnicas para incremento de produtividade e a rentabilidade das culturas. Contudo, há poucas informações de informações sobre a resposta da respectiva cultivar a adubação química com $\mathrm{N}-\mathrm{P}_{2} 05-\mathrm{K}_{2} \mathrm{O}$. Além disso, existem poucos relatos sobre o comportamento da mamoneira sob diferentes condições de fertilidade do solo, clima e disponibilidade de água. Essa cultura é exigente em fertilidade, sendo possível aumentar sua produtividade pelo adequado fornecimento de nutrientes pela fertilização do solo (MESQUITA, 2010).

Dessa forma atribui-se esses resultados de significância para a casca natural devido o decorrer do tempo, já que a sua mineralização ocorre de forma gradual, proporcionado conseqüentemente a distribuição dos nutrientes lentamente para a planta.Outro fator é a presença em teor considerável do nutriente $\mathrm{K}$ na casca de mamona.

Ilustra-se na figura 3 uma influência significativa da aplicação de doses crescentes de N no comportamento produtivo da mamoneira para a variável número de sementes por cacho (NSCH) em função de diferentes doses de nitrogênio. Observa-se que houveum aumento proporcional na produção, obtendo-se o melhor resultado para a dosagem de $30 \mathrm{~kg} / \mathrm{ha}^{-1}$, logo em seguida observa-se um declínio na produção para dosagem de $60 \mathrm{~kg} \mathrm{ha}^{-1}$ 


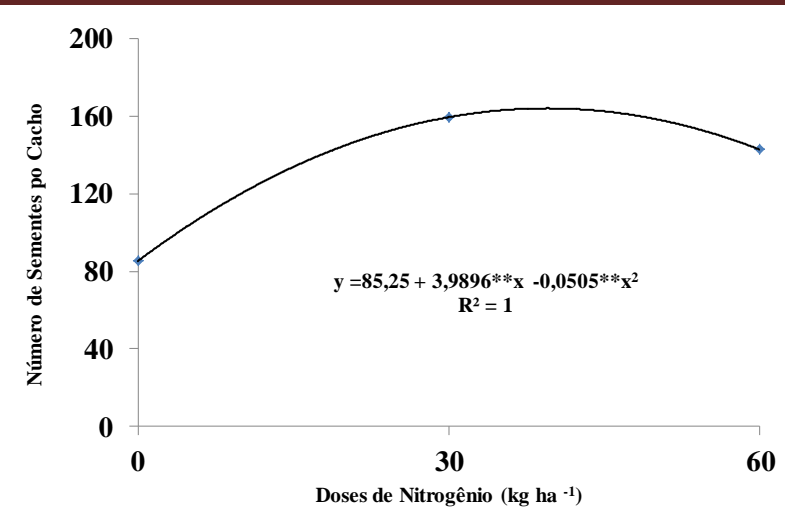

Figura 3. Modelos de regressão para o número de sementes do cacho da mamoneira BRS Energia em função de doses crescentes de Nitrogênio. Catolé do Rocha - PB, 2011.

A ilustração 4 mostra o comportamento da fitomassa de 100 sementes do cacho em função de diferentes doses de nitrogênio,obtiveram-se efeito significativo e observou-se que nenhuma das variáveis estudadas sofreu efeito significativo para fator tipo de casca.

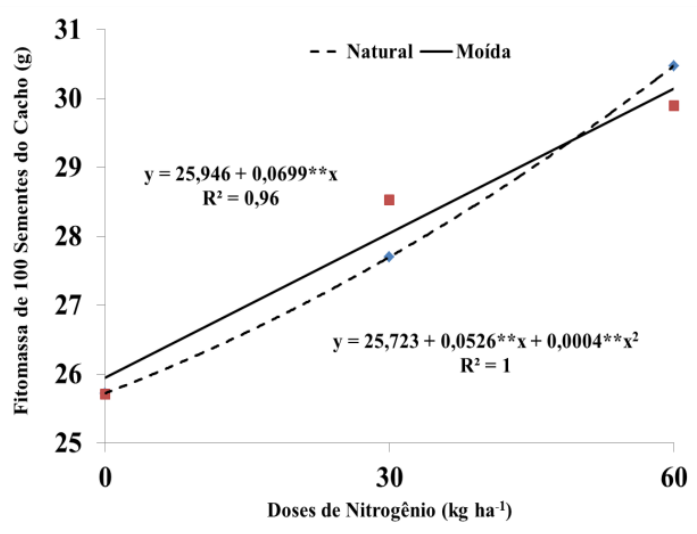

Figura 4. Modelos de regressão para a fitomassa de 100 sementes do cacho da mamoneira BRS Energia em função de doses crescentes de Nitrogênio. Catolé do Rocha - PB, 2011.

O presente estudo encontra respaldo em estudos realizados por Guareschi et al. (2008), estudando a produção de massa de milho silagem em função do arranjo populacional e adubação, assim, como também Santos et al. (2010), estudando efeito residual da adubação orgânica sobre a produtividade de milho em sistema agroflorestal. Por outro lado, Pimentel et al. (2009), trabalhando com rendimentos agronômicos em consórcio de alface e cenoura adubadas com doses crescentes de composto orgânico, constataram que na cultura da alface, o diâmetro da parte aérea e o teor de massa seca, responderam aos tratamentos com composto orgânicos, enquanto na cultura da cenoura, a produtividade. Tendo o aumento da dose de composto orgânico proporcionado redução do teor de massa seca, tanto de raízes de cenoura, quanto da parte aérea da alface. 
Lima et al (2008) em estudos com casca e torta de mamona avaliados em vasos como fertilizantes orgânicos, relata que quando a casca de mamona é usada na adubação em vaso, é preciso que antes seja submetido a um processo de compostagem, decomposição ou misturada a outro material rico em $\mathrm{N}$, o que reduz a relação $\mathrm{C} / \mathrm{N}$ da mesma.

O presente estudo demonstra que a mamoneira responde a fertilização orgânica, a qual fornece nutriente, melhora as características físicas e químicas do solo, no entanto não é suficiente, deve ser complementada com a adubação mineral que estabeleça fatores favoráveis para uma determinada cultura.

\section{Conclusões}

1. Os maiores valores obtidos foi com a dose de $60 \mathrm{~kg} / \mathrm{ha}^{-1} \mathrm{de} \mathrm{N}$, superando respectivamente as demais quantidades de 30 e $0 \mathrm{~kg} / \mathrm{ha}^{-1}$ de $\mathrm{N}$.

2. Para os resultados das variáveis de produção estudadas, indicaram que a fitomassa do caule, fitomassa da raiz principal, número de sementes do cacho e a fitomassa de 100 sementes do cacho em função de diferentes doses de nitrogênio obtiveram efeitos significativos.

3. Observou-se que nenhuma das variáveis de produção estudadas sofreu efeitos significativos na adubação orgânica para fator tipo de casca.

\section{Agradecimentos}

Agradecemos a Universidade Estadual da Paraíba e Embrapa Algodão por todo apoio científico e financeiro para realização deste experimento.

\section{Referências}

BAYER, C.; MIELNICZUK. Macromoléculas e substâncias húmicas. In: SANTOS, G. A.; SILVA, L.S.; CANELLAS, L. P.; CAMARGO, F. A. O. (Eds). Fundamentos da matéria orgânica do solo: Ecossistemas tropicais e subtropicais. Porto Alegre: RS, p.7 - 162008.

Beltrão, N. E. de M.; Vale, L. S. Do.; Silva, o. R. R. F. da. Agricultura Tropical: Quatro Décadas de Inovações Tecnológicas, Institucionais e Políticas. Vol. 1. Produção e 
produtividade Agrícola. In: Grãos oleaginosos. Cap. 4. Brasília: EMBRAPA Informação Tecnológica. 2008.

Costa, F. X.; Beltrão, N. E. M.; Lima, V. L. A. DE; Nunes Junior, E. S; Guimarães, M. M. B.; Damaceno, F. A. V. Efeito do lixo orgânico e torta de mamona nas características de crescimento da mamoneira (Ricinus Communis L.). Revista Engenharia com Ambiental: pesquisa e tecnologia Espírito Santo do Pinhal, v. 6, n. 1, p. 259-268, jan/abr 2009.

Coelho, D. K. Efeitos na mamoneira da irrigação com águas salinas e adubação com polpa de mamona. Campina Grande - PB, UFCG, 2006, 89 p. Dissertação de Mestrado.

Filho, J. S.; Costa, F. X; Nunes Junior, E. S; Silva, F. E. A.; Silva, M. A. Produção da mamoneira em Função do efeito Residual da Adubação Orgânica. Revista Verde de Agroecologia e Desenvolvimento Sustentável: Mossoró-RN-Brasil, v. 7, n. 1, p. 116-121, jan/mar 2012.

Guareschi, R. F.; Gazolla, P.R.; Perin, A.; Rocha, A. C. da.Produção de massa de milho silagem em função do arranjo populacional e adubação. Revista Ciência Agronômica, v. 39, n. 3, p. 468-475, jul-set, 2008.

LAVRES JUNIOR, J.; NOGUEIRA, T. A. R.; CABRAL, C. P.; MALAVOLTA, E.. Deficiências de macronutrientes no crescimento e na produção da mamoneira cultivar Íris. Revista Brasileira de Ciências Agrárias, v.4, n.4, p.405- 413, 2009.

Lima, E. F. S.; Severino, L. S.; Albuquerque, R.C.; Beltrão, N. E. M.; Sampaio, L. R. Casca e torta de mamona avaliados em vasos como fertilizantes orgânicos. Revista Caatinga, v.21, n.5, p.102-106, 2008.

Lima, E. F. S.; Severino, L. S.; Silva, M. I. L.; Vale L. S. Crescimento inicial de mudas de mamoneira em substrato contendo lodo de esgoto e casca de amendoim. Revista brasileira de oleaginosas e fibrosas. v.9, n.1/3, p.887-891, jan/dez,

MARENCO, R. A.; LOPES, N. F. Fisiologia Vegetal. Viçosa: 3 ed, UFV, 486 p., 2009.

MEDEIROS, J.F: SILVA, M.C.C.; SARMENTO D. H.A.; BARROS, A. D. Crescimento do meloeiro cultivado sob diferentes níveis de salinidade, com e sem cobertura do solo. Revista Brasileira de Engenharia Agrícola e Ambiental, Campina Grande v. 11, n.3, p. 248-255, 2007.

MESQUITA, E. F. Comportamento de duas cultivares de mamona irrigada sob fertilização do solo com NPK. 2010108 f. Tese (Doutorado em Engenharia Agrícola) Universidade Federal de Campina Grande, Campina Grande. 2010.

MORO, E. Manejo da adubação nitrogenada em híbridos de mamona de porte baixo cultivados na safra e na safrinha em sistema plantio direto. 2008. 118 f. Dissertação (Mestrado em Agronomia) - Universidade Estadual de Paulista, Botucatu. 2008.

Pimentel, M. S.; Lana, Â. M. Q.; De-Polli, H. Rendimentos agronômicos em consórcio de alface e cenoura adubadas com doses crescentes de composto orgânico. Revista Ciência Agronômica, v. 40, n. 1, p. 106-112, jan-mar, 2009. 
PROCHNOW, L. I.; ROSSI, F. Análise de solo e recomendação de calagem e adubação.Viçosa: CPT, 308 p., 2009.

Santos, A. F. DOS; Menezes, R. S. C.; Fraga, V. S.; Pérez-Marin, A. M. Efeito residual da adubação orgânica sobre a produtividade de milho em sistema agroflorestal. Revista Brasileira de Engenharia Agrícola e Ambiental, v.14, n.12, p.1267-1272, 2010.

Severino, L. S.; Lima, R. L. S. de; Albuquerque, R. C., Beltrão, N. E. M. Mamona: O produtor pergunta, a Embrapa responde. Brasília, DF: Embrapa Informação Tecnológica; Campina Grande - PB: EMBRAPA Algodão, 2006. (Coleção 500 perguntas, 500 respostas).

Severino, L. S.; Ferreira, G. B.; Moraes, C. R. A.; Gondin, T. M. S.; Freire, W. S. A.; Castro, D. A.; Cardoso, G. D.; Beltrão, N. E. M. Adubação Química da Mamoneira com Macro e Micronutrientes em Quixeramobim, CE. Campina Grande, PB. Embrapa Algodão, 2005. 23 p. (Embrapa Algodão. Boletim de Pesquisa e Desenvolvimento, 61).

Silva, N.G. A; Lino, A.S.MAMONA e biodiesel: oportunidade para o semi-árido. Disponível em: http::Iwww.sober.org.br palestra (9) 341pdf.Acesso em:30 abr.2009.

SOUZA, L. A.; CARVALHO, M. L. M.; KATAOKA, V. Y.; OLIVEIRA, J. A. Teste de condutividade elétrica para avaliação da qualidade fisiológica de sementes de mamona.

Revista Brasileira de Sementes, Lavras, v.31, n.1, p. 60-67, 2009. 